\title{
Development of Geant4 based simulation for Super-Kamiokande
}

\author{
M.Harada*1, Y.Koshio ${ }^{1}$, K.Hagiwara ${ }^{1}$ \\ ${ }^{1}$ Okayama University, Okayama-shi, Okayama, 700-8530, Japan \\ ${ }^{*}$ E-mail: pc3a4sejes.okayama-u.ac.jp
}

\begin{abstract}
Currently, Super-K experiment uses Geant3 based simulation software (SKDETSIM), and the new phase of the experiment, which is called "SK-Gd Project" will start soon. Main purpose of the SK-Gd is the first discovery of the Diffuse Supernova Neutrino Background, which is neutrinos generated from the past Supernova explosion. In SK-Gd, precise understanding about neutron capture of Gadolinium is crucial. In order to apply the latest interaction model based on the new experimental results, Geant4 based Super-K simulation software "SKG4" is now on developing. In this article, The current development status of SKG4, and some results of comparison with SKDETSIM are presented.
\end{abstract}

The 4th KMI International Symposium (KMI2019)

18-20, February 2019

Nagoya, Japan

${ }^{*}$ Speaker. 


\section{Introduction}

Neutrinos emitted from past core-collapse supernova (CCSN) are expected to drift in our universe. This is often called Diffuse Supernova Neutrino Background(DSNB). Detection of DSNB would give a new insight about the history of star formation and theory of supernova. SuperKamiokande (SK) experiment is aiming to observe these neutrinos. All flavors of neutrino are emitted from CCSN. Among them, anti-electron neutrino has the largest cross section via inverse beta decay (IBD) reaction $\left(\bar{v}_{e}+p \rightarrow e^{+}+n\right)$ in the energy range around few tens $\mathrm{MeV}$ that SK focuses DSNB. A search for DSNB using positron signal was carried out in SK. As a result, although DSNB signal couldn't detect, an upper limit of DSNB flux for $E_{\bar{v}_{e}}>17.3 \mathrm{MeV}$ was derived[四]. The search was suffered from some background. The most serious background of current DSNB search is decay electron from invisible muon, which is produced by atmospheric neutrinos. This electron signal is not possible to be distinguished with positron signal from DSNB in Water-Cherenkov detector.

To separate the DSNB signal from many background channel, further search with lower energy threshold, which used delayed neutron signal $\left(E_{\bar{v}_{e}}>13.3 \mathrm{MeV}\right)$ was carried out[[]]. $2.2 \mathrm{MeV}$ gamma ray from neutron capture reaction of proton is used as the delayed neutron signal. However, the delayed neutron signal is very low energy for SK, so that detection efficiency of delayed neutron signal was less than $20 \%$ and the limit of DSNB flux which is derived in this search is worse than previous search[四].

To observe DSNB, it is needed that the further reduction of background and the expansion of energy region in search. Therefore, SuperKamiokande-Gadolinium project(SK-Gd) is in preparation[3]. Gd has the highest thermal neutron capture cross section among all stable elements and the gamma-rays are emitted from neutron capture reaction are totally $\sim 8 \mathrm{MeV}$ which can be easily detected by SK. SK-Gd enables detection of neutron signal with high detection efficiency by dissolving $0.2 \% \mathrm{Gd}_{2}\left(\mathrm{SO}_{4}\right)_{3}$ in mass concentration in SK. Figure 1 shows the detection method of IBD by using prompt positron signal and delayed neutron signal. Toward SK-Gd, SK tank refurbishment work was completed in 2018, and pure water operation has already started since the end of January 2019.

\section{Super-K Simulation}

Current SK detector simulation(SKDETSIM) is based on Geant3[四]. There are some problems to use SKDETSIM in future. The most serious problem is that the update of physics model in Geant 3 is already stopped. Therefore, SKDETSIM has poor extensibility, and some physics models may be inaccurate. Especially, neutron interaction model is crucial for SK-Gd.

To improve these problems, new detector simulation software based on Geant4[[]] was made, this is named "SKG4". SKG4 uses the latest physics model which is suitable for SK, especially, the model of gamma ray which is emitted from neutron capture on $\mathrm{Gd}$ is based on very accurate experimental result [鴫. Due to these advantages, SKG4 will be possible to perform highly accurate simulation on SK-Gd.

\section{Check the physics process}

The reproduction of SK geometry was already completed. After that, physics process must be checked. Several checks has been performed as described below. 


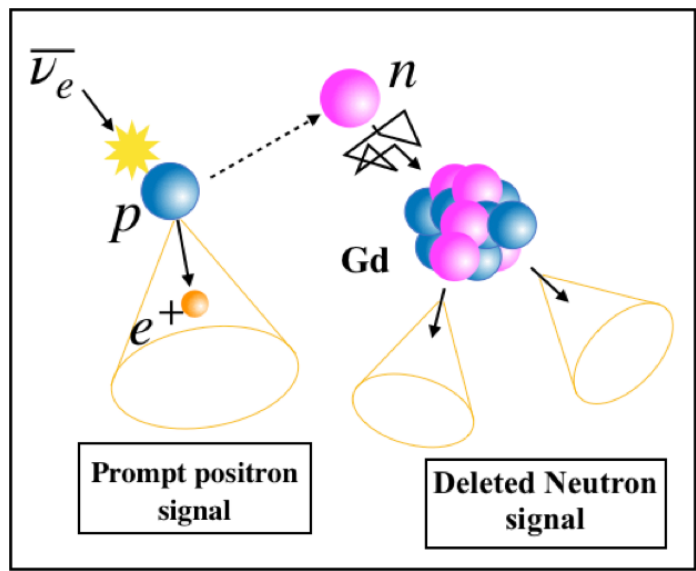

Figure 1: Schematic of inverse beta decay in SK-Gd. It will become to be possible to "Delayed coincidence" measurement.

\subsection{Electromagnetic(EM) physics}

Electromagnetic process is one of the most important for SK physics. SKG4 adopt the Livermore physics list $[\square]$. By generating $10 \mathrm{MeV}$ electron from the center of the tank, the number of emitted Cherenkov photon and hit on PMT is compared with SKDETSIM. Any optical property of photon is not considered in this situation. Table 1 shows the result of both simulation. This difference comes from the algorithm of Cherenkov photon emission. Even if there is a few percent difference, finally, it will be tuned by calibration data in SK.

\begin{tabular}{c|c|c} 
& Number of generated photons & Number of Photons which hit PMT \\
\hline SKDETSIM & 1948 & 760 \\
SKG4 & 1899 & 740 \\
\hline \hline Ratio & $2.6 \%$ & $2.6 \%$
\end{tabular}

Table 1: Comparison of cherenkov photon emitted from $10 \mathrm{MeV}$ electron between SKG4 and SKDETSIM.

\subsection{Optical photon physics}

Optical photon has some kinds of process. Figure 2 shows the rate of decrease in the mean of number of hits due to the absorption effect in water for Cherenkov photons emitted from $10 \mathrm{MeV}$ electrons. The decrease due to absorption was in good agreement at $0.2 \%$ with SKDETSIM.

\subsection{Gd neutron capture process}

Figure 3 shows the gamma ray spectrum which is emitted from neutron capture on Gd and proton. It was confirmed that the gamma ray energy have a distribution until around $8 \mathrm{MeV}$. Table 2 shows the neutron capture time and distance, and probability of captured by each nuclei for each situation(pure water, $0.2 \% \mathrm{Gd}_{2}\left(\mathrm{SO}_{4}\right)_{3}$ dissolved water). These values were obtained from the simulation of the neutrons generated from center of the tank. 


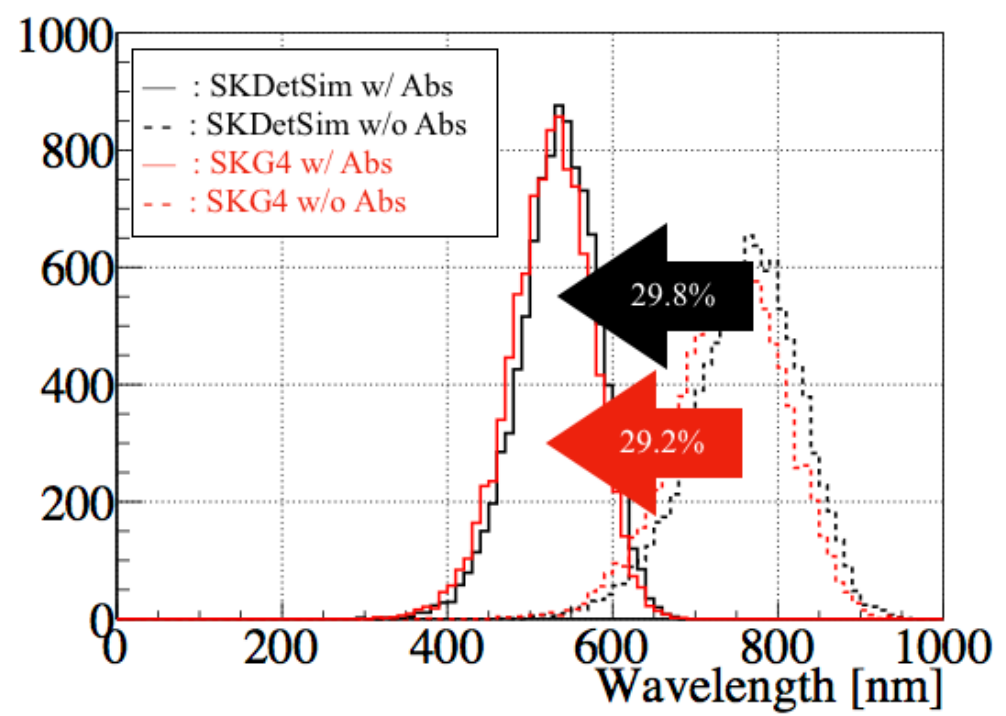

Figure 2: Number of PMT hit with(solid line) or without(dashed line) absorption of water. Red line shows SKG4, black line shows SKDETSIM.The mean value of the distribution is decreased about $29 \%$

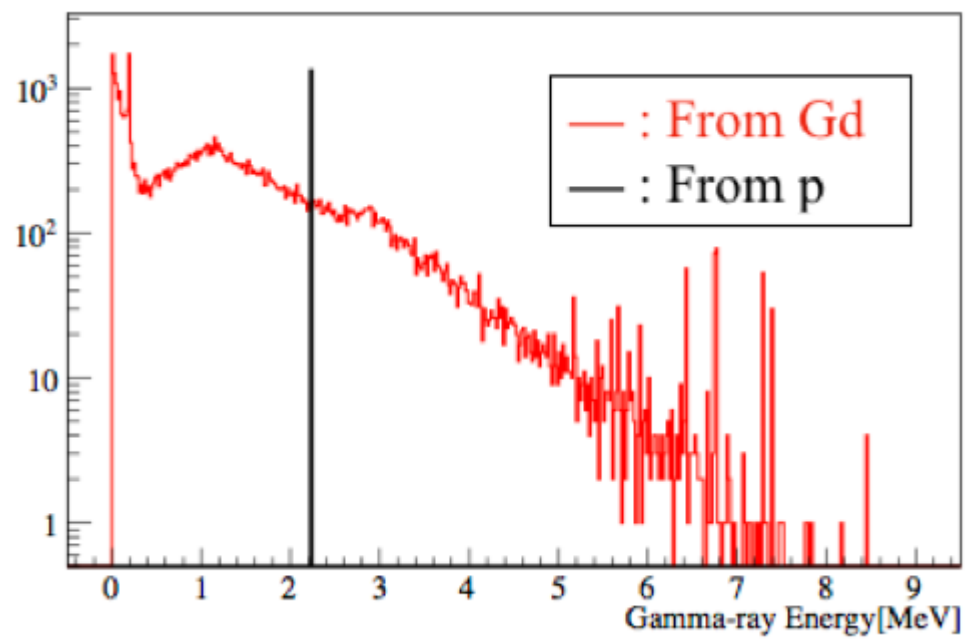

Figure 3: Energy spectrum of gamma ray from neutron capture by Gd or proton. Red line shows captured by $\mathrm{Gd}$, black line shows proton.

\begin{tabular}{c|c|c|c} 
& Captured time & Distance & Probability \\
\hline Captured by proton(Pure water) & $196 \pm 3 \mu \mathrm{s}$ & $107 \pm 60 \mathrm{~mm}$ & - \\
Captured by proton $\left(0.2 \% \mathrm{Gd}_{2}\left(\mathrm{SO}_{4}\right)_{3}\right.$ dissolved water $)$ & $18 \pm 1 \mu \mathrm{s}$ & $68 \pm 31 \mathrm{~mm}$ & $13.27 \pm 0.36 \%$ \\
Captured by $\mathrm{Gd}\left(0.2 \% \mathrm{Gd}_{2}\left(\mathrm{SO}_{4}\right)_{3}\right.$ dissolved water $)$ & $27 \pm 1 \mu \mathrm{s}$ & $70 \pm 33 \mathrm{~mm}$ & $86.7 \pm 0.93 \%$
\end{tabular}

Table 2: Calculated capture time, distance, probability of $200 \mathrm{keV}$ neutron by each neuclei in Gd dissolved water or pure water. 
It is found that the time and distance from prompt signal become shorter by dissolving $\mathrm{Gd}$, and the probability of capture on Gd is about $90 \%$. This is good agreement with the expectation as written in ref [B]].

\section{Conclusion and Status}

SK-Gd experiment will start in 2019. Toward SK-Gd, new simulation software named SKG4 is constructed. Geometry of SK already reproduces with the current simulation, SKDETSIM. In this article, some behavior of physics in SKG4 is compared SKDETSIM. The difference of number of emitted Cherenkov photon by $10 \mathrm{MeV}$ electron with SKDETSIM is 2.6\%. Difference of reduction ratio by absorption effect from SKDETSIM is $0.2 \%$. New model about gamma ray from $\mathrm{Gd}(\mathrm{n}, \gamma)$ reaction is also checked. It is found that neutron capture time and distance from the position where neutron capture occurred is shorter than pure water situation. At present, comparison and tuning by using real SK calibration data is in progress. After dissolving Gd in SK water, SKG4 will be used various analysis in SK-Gd.

\section{Acknowledgments}

We gratefully acknowledge cooperation of the Kamioka Mining and Smelting Company. The Super-Kamiokande experiment was built and has been operated with funding from the Japanese Ministry of Education, Science, Sports and Culture, and the U.S. Department of Energy.

\section{References}

[1] K. Bays et al., Phys. Rev. D 85, 052007 (2012).

[2] H. Zhang et al., Astropart. Phys. 60, 41-46 (2015), arXiv:1311.3738.

[3] J. F. Beacom and M. R. Vagins, Phys. Rev. Lett., 93, 171101 (2004).

[4] R. Brun, F. Bruyant, M. Maire, A. C. McPherson and P. Zanarini, CERN-DD-EE-84-1 (1987).

[5] S. Agostinelli et al., Nucl. Instrum, Meth. A506 (2003).

[6] K.Hagiwara et al., Prog. Theor. Exp. Phys. (2018), arXiv:1809.02664v1.

[7] J. Apostolakis et al., Technical Report, CERN/INFN, CERN-OPEN-99-034(1999), INFN/AE-99/18. 